\title{
UPPER BOUNDS FOR NUMERICAL RADIUS INEQUALITIES INVOLVING OFF-DIAGONAL OPERATOR MATRICES
}

\author{
MOJTABA BAKHERAD ${ }^{1}$ AND KHALID SHEBRAWI ${ }^{2}$
}

\begin{abstract}
In this paper, we establish some upper bounds for numerical radius inequalities including of $2 \times 2$ operator matrices and their off-diagonal parts. Among other inequalities, it is shown that if $T=\left[\begin{array}{cc}0 & X \\ Y & 0\end{array}\right]$, then$$
\omega^{r}(T) \leq 2^{r-2}\left\|f^{2 r}(|X|)+g^{2 r}\left(\left|Y^{*}\right|\right)\right\|^{\frac{1}{2}}\left\|f^{2 r}(|Y|)+g^{2 r}\left(\left|X^{*}\right|\right)\right\|^{\frac{1}{2}}
$$

and

$$
\omega^{r}(T) \leq 2^{r-2}\left\|f^{2 r}(|X|)+f^{2 r}\left(\left|Y^{*}\right|\right)\right\|^{\frac{1}{2}}\left\|g^{2 r}(|Y|)+g^{2 r}\left(\left|X^{*}\right|\right)\right\|^{\frac{1}{2}},
$$

where $X, Y$ are bounded linear operators on a Hilbert space $\mathscr{H}, r \geq 1$ and $f, g$ are nonnegative continuous functions on $[0, \infty)$ satisfying the relation $f(t) g(t)=t(t \in$ $[0, \infty))$. Moreover, we present some inequalities involving the generalized Euclidean operator radius of operators $T_{1}, \cdots, T_{n}$.

\section{INTRODUCTION}

Let $\mathbb{B}(\mathscr{H})$ denote the $C^{*}$-algebra of all bounded linear operators on a Hilbert space $\mathscr{H}$. In the case when $\operatorname{dim} \mathscr{H}=n$, we identify $\mathbb{B}(\mathscr{H})$ with the matrix algebra $\mathbb{M}_{n}$ of all $n \times n$ matrices with entries in the complex field. An operator $A \in \mathbb{B}(\mathscr{H})$ is said to be contraction, if $A^{*} A \leq I$. The numerical radius of $T \in \mathbb{B}(\mathscr{H})$ is defined by

$$
\omega(T):=\sup \{|\langle T x, x\rangle|: x \in \mathscr{H},\|x\|=1\} .
$$

It is well known that $\omega(\cdot)$ defines a norm on $\mathbb{B}(\mathscr{H})$, which is equivalent to the usual operator norm. In fact, $\frac{1}{2}\|\cdot\| \leq \omega(\cdot) \leq\|\cdot\|$; see [9]. An important inequality for $\omega(A)$ is the power inequality stating that $\omega\left(A^{n}\right) \leq \omega(A)^{n}(n=1,2, \cdots)$. For further information about the properties of numerical radius inequalities we refer the reader to $[1,5,13]$ and references therein. Let $\mathscr{H}_{1}, \mathscr{H}_{2}$ be Hilbert spaces, and consider the direct sum $\mathscr{H}=\mathscr{H}_{1} \oplus \mathscr{H}_{2}$. With respect to this decomposition, every operator $T \in \mathbb{B}(\mathscr{H})$ has a $2 \times 2$ operator matrix representation $T=\left[T_{i j}\right]$ with entries $T_{i j} \in \mathbb{B}\left(\mathscr{H}_{j}, \mathscr{H}_{i}\right)$, the

2010 Mathematics Subject Classification. Primary 47A12, Secondary 47A30, 47A63, 47B33.

Key words and phrases. numerical radius; off-diagonal part; positive operator; Young inequality; generalized Euclidean operator radius. 
space of all bounded linear operators from $\mathscr{H}_{j}$ to $\mathscr{H}_{i}(1 \leq i, j \leq 2)$. Operator matrices provide a usual tool for studying Hilbert space operators, which have been extensively studied in the literatures. Let $A \in \mathbb{B}\left(\mathscr{H}_{1}, \mathscr{H}_{1}\right), B \in \mathbb{B}\left(\mathscr{H}_{2}, \mathscr{H}_{1}\right), C \in \mathbb{B}\left(\mathscr{H}_{1}, \mathscr{H}_{2}\right)$ and $D \in \mathbb{B}\left(\mathscr{H}_{2}, \mathscr{H}_{2}\right)$. The operator $\left[\begin{array}{cc}A & 0 \\ 0 & D\end{array}\right]$ is called the diagonal part of $\left[\begin{array}{cc}A & B \\ C & D\end{array}\right]$ and $\left[\begin{array}{cc}0 & B \\ C & 0\end{array}\right]$ is the off-diagonal part.

The classical Young inequality says that if $p, q>1$ such that $\frac{1}{p}+\frac{1}{q}=1$, then $a b \leq \frac{a^{p}}{p}+\frac{b^{q}}{q}$ for positive real numbers $a, b$. In [3], the authors showed that a refinement of the scalar Young inequality as follows $\left(a^{\frac{1}{p}} b^{\frac{1}{q}}\right)^{m}+r_{0}^{m}\left(a^{\frac{m}{2}}-b^{\frac{m}{2}}\right)^{2} \leq\left(\frac{a}{p}+\frac{b}{q}\right)^{m}$, where $r_{0}=\min \left\{\frac{1}{p}, \frac{1}{q}\right\}$ and $m=1,2, \cdots$. In particular, if $p=q=2$, then

$$
\left(a^{\frac{1}{2}} b^{\frac{1}{2}}\right)^{m}+\left(\frac{1}{2}\right)^{m}\left(a^{\frac{m}{2}}-b^{\frac{m}{2}}\right)^{2} \leq 2^{-m}(a+b)^{m} .
$$

It has been shown in [8], that if $T \in \mathbb{B}(\mathscr{H})$, then

$$
\omega(T) \leq \frac{1}{2}\left\||T|+\left|T^{*}\right|\right\|,
$$

where $|T|=\left(T^{*} T\right)^{\frac{1}{2}}$ is the absolute value of $T$. Recently [2], the authors extended this inequality for off-diagonal operator matrices of the form $T=\left[\begin{array}{cc}0 & X \\ Y & 0\end{array}\right] \in \mathbb{B}\left(\mathscr{H}_{1} \oplus \mathscr{H}_{2}\right)$ as follows

$$
\omega(T) \leq \frac{1}{2}\left\||X|+\left|Y^{*}\right|\right\|^{\frac{1}{2}}\left\|\left|X^{*}\right|+|Y|\right\|^{\frac{1}{2}} .
$$

Let $T_{1}, T_{2}, \cdots, T_{n} \in \mathbb{B}(\mathscr{H})$. The functional $\omega_{p}$ of operators $T_{1}, \cdots, T_{n}$ for $p \geq 1$ is defined in [11] as follows

$$
\omega_{p}\left(T_{1}, \cdots, T_{n}\right):=\sup _{\|x\|=1}\left(\sum_{i=1}^{n}\left|\left\langle T_{i} x, x\right\rangle\right|^{p}\right)^{\frac{1}{p}} .
$$

If $p=2$, then we have the Euclidean operator radius of $T_{1}, \cdots, T_{n}$ which was defined in [10]. In [13], the authors showed that an upper bound for the functional $\omega_{p}$

$$
\omega_{p}^{p}\left(T_{1}, \cdots, T_{n}\right) \leq \frac{1}{2}\left\|\sum_{i=1}^{n}\left(f^{2 p}\left(\left|T_{i}\right|\right)+g^{2 p}\left(\left|T_{i}^{*}\right|\right)\right)\right\|-\inf _{\|x\|=1} \zeta(x),
$$

where $T_{i} \in \mathbb{B}(\mathscr{H})(i=1,2, \cdots, n), f, g$ are nonnegative continuous functions on $[0, \infty)$ such that $f(t) g(t)=t(t \in[0, \infty)), p \geq 1$ and

$$
\zeta(x)=\frac{1}{2} \sum_{i=1}^{n}\left(\left\langle f^{2 p}\left(\left|T_{i}\right|\right) x, x\right\rangle^{\frac{1}{2}}-\left\langle g^{2 p}\left(\left|T_{i}^{*}\right|\right) x, x\right\rangle^{\frac{1}{2}}\right)^{2} .
$$


In this paper, we show some inequalities involving powers of the numerical radius for off-diagonal parts of $2 \times 2$ operator matrices. In particular, we extend inequalities (1.2) and (1.3) for nonnegative continuous functions $f, g$ on $[0, \infty)$ such that $f(t) g(t)=t(t \in$ $[0, \infty))$. Moreover, we present some inequalities including the generalized Euclidean operator radius $\omega_{p}$.

\section{MAIN RESULTS}

To prove our first result, we need the following lemmas.

Lemma 2.1. [6, 14] Let $X \in \mathbb{B}(\mathscr{H})$. Then

$$
\begin{aligned}
& \text { (a) } \omega(X)=\max _{\theta \in \mathbb{R}}\left\|\operatorname{Re}\left(e^{i \theta} X\right)\right\|=\max _{\theta \in \mathbb{R}}\left\|\operatorname{Im}\left(e^{i \theta} X\right)\right\| . \\
& \text { (b) } \omega\left(\left[\begin{array}{cc}
0 & X \\
X & 0
\end{array}\right]\right)=\omega(X) .
\end{aligned}
$$

The next lemma follows from the spectral theorem for positive operators and Jensen inequality; see [7].

Lemma 2.2. Let $T \in \mathbb{B}(\mathscr{H}), T \geq 0$ and $x \in \mathscr{H}$ such that $\|x\| \leq 1$. Then

(a) $\langle T x, x\rangle^{r} \leq\left\langle T^{r} x, x\right\rangle$ for $r \geq 1$.

(b) $\left\langle T^{r} x, x\right\rangle \leq\langle T x, x\rangle^{r}$ for $0<r \leq 1$.

Proof. Let $r \geq 1$ and $x \in \mathscr{H}$ such that $\|x\| \leq 1$. Fix $u=\frac{x}{\|x\|}$. Using the McCarty inequality we have $\langle T u, u\rangle^{r} \leq\left\langle T^{r} u, u\right\rangle$, whence

$$
\begin{aligned}
\langle T x, x\rangle^{r} & \leq\|x\|^{2 r-2}\left\langle T^{r} x, x\right\rangle \\
& \leq\left\langle T^{r} x, x\right\rangle \quad(\text { since }\|x\| \leq 1 \text { and } 2 r-2 \geq 0) .
\end{aligned}
$$

Hence, we get the first inequality. The proof of the second inequality is similar.

Lemma 2.3. [7, Theorem 1] Let $T \in \mathbb{B}(\mathscr{H})$ and $x, y \in \mathscr{H}$ be any vectors. If $f, g$ are nonnegative continuous functions on $[0, \infty)$ which are satisfying the relation $f(t) g(t)=$ $t(t \in[0, \infty))$, then

$$
|\langle T x, y\rangle|^{2} \leq\left\langle f^{2}(|T|) x, x\right\rangle\left\langle g^{2}\left(\left|T^{*}\right|\right) y, y\right\rangle .
$$


Now, we are in position to demonstrate the main results of this section by using some ideas from $[2,13]$.

Theorem 2.4. Let $T=\left[\begin{array}{cc}0 & X \\ Y & 0\end{array}\right] \in \mathbb{B}\left(\mathscr{H}_{1} \oplus \mathscr{H}_{2}\right), r \geq 1$ and $f, g$ be nonnegative continuous functions on $[0, \infty)$ satisfying the relation $f(t) g(t)=t(t \in[0, \infty))$. Then

$$
\omega^{r}(T) \leq 2^{r-2}\left\|f^{2 r}(|X|)+g^{2 r}\left(\left|Y^{*}\right|\right)\right\|^{\frac{1}{2}}\left\|f^{2 r}(|Y|)+g^{2 r}\left(\left|X^{*}\right|\right)\right\|^{\frac{1}{2}}
$$

and

$$
\omega^{r}(T) \leq 2^{r-2}\left\|f^{2 r}(|X|)+f^{2 r}\left(\left|Y^{*}\right|\right)\right\|^{\frac{1}{2}}\left\|g^{2 r}(|Y|)+g^{2 r}\left(\left|X^{*}\right|\right)\right\|^{\frac{1}{2}} .
$$

Proof. Let $\mathbf{x}=\left[\begin{array}{l}x_{1} \\ x_{2}\end{array}\right] \in \mathscr{H}_{1} \oplus \mathscr{H}_{2}$ be a unit vector (i.e., $\left\|x_{1}\right\|^{2}+\left\|x_{2}\right\|^{2}=1$ ). Then

$$
\begin{aligned}
& |\langle T \mathbf{x}, \mathbf{x}\rangle|^{r} \\
& =\left|\left\langle X x_{2}, x_{1}\right\rangle+\left\langle Y x_{1}, x_{2}\right\rangle\right|^{r} \\
& \leq\left(\left|\left\langle X x_{2}, x_{1}\right\rangle\right|+\left|\left\langle Y x_{1}, x_{2}\right\rangle\right|\right)^{r} \quad \text { (by the triangular inequality) } \\
& \left.\leq \frac{2^{r}}{2}\left(\left|\left\langle X x_{2}, x_{1}\right\rangle\right|^{r}+\left|\left\langle Y x_{1}, x_{2}\right\rangle\right|^{r}\right) \quad \text { (by the convexity } f(t)=t^{r}\right) \\
& \leq \frac{2^{r}}{2}\left(\left(\left\langle f^{2}(|X|) x_{2}, x_{2}\right\rangle^{\frac{1}{2}}\left\langle g^{2}\left(\left|X^{*}\right|\right) x_{1}, x_{1}\right\rangle^{\frac{1}{2}}\right)^{r} \quad \quad\right. \text { (by Lemma 2.3) } \\
& \left.\quad+\left(\left\langle f^{2}(|Y|) x_{1}, x_{1}\right\rangle^{\frac{1}{2}}\left\langle g^{2}\left(\left|Y^{*}\right|\right) x_{2}, x_{2}\right\rangle^{\frac{1}{2}}\right)^{r}\right) \quad x^{r} \quad\left(\left\langle f^{2 r}(|X|) x_{2}, x_{2}\right\rangle^{\frac{1}{2}}\left\langle g^{2 r}\left|X^{*}\right| x_{1}, x_{1}\right\rangle^{\frac{1}{2}}+\left\langle f^{2 r}(|Y|) x_{1}, x_{1}\right\rangle^{\frac{1}{2}}\left\langle g^{2 r}\left(\left|Y^{*}\right|\right) x_{2}, x_{2}\right\rangle^{\frac{1}{2}}\right) \\
& \leq \frac{2^{r}}{2}
\end{aligned}
$$

(by Lemma 2.2(a))

$\leq \frac{2^{r}}{2}\left(\left\langle f^{2 r}(|X|) x_{2}, x_{2}\right\rangle+\left\langle g^{2 r}\left(\left|Y^{*}\right|\right) x_{2}, x_{2}\right\rangle\right)^{\frac{1}{2}}$

$\times\left(\left\langle f^{2 r}(|Y|) x_{1}, x_{1}\right\rangle+\left\langle g^{2 r}\left(\left|X^{*}\right|\right) x_{1}, x_{1}\right\rangle\right)^{\frac{1}{2}} \quad$ (by the Cauchy-Schwarz inequality)

$=\frac{2^{r}}{2}\left\langle\left(f^{2 r}(|X|)+g^{2 r}\left(\left|Y^{*}\right|\right)\right) x_{2}, x_{2}\right\rangle^{\frac{1}{2}}\left\langle\left(f^{2 r}(|Y|)+g^{2 r}\left(\left|X^{*}\right|\right)\right) x_{1}, x_{1}\right\rangle^{\frac{1}{2}}$

$\leq \frac{2^{r}}{2}\left\|f^{2 r}(|X|)+g^{2 r}\left(\left|Y^{*}\right|\right)\right\|^{\frac{1}{2}}\left\|f^{2 r}(|Y|)+g^{2 r}\left(\left|X^{*}\right|\right)\right\|^{\frac{1}{2}}\left\|x_{1}\right\|\left\|x_{2}\right\|$

$\leq \frac{2^{r}}{2}\left\|f^{2 r}(|X|)+g^{2 r}\left(\left|Y^{*}\right|\right)\right\|^{\frac{1}{2}}\left\|f^{2 r}(|Y|)+g^{2 r}\left(\left|X^{*}\right|\right)\right\|^{\frac{1}{2}}\left(\frac{\left\|x_{1}\right\|^{2}+\left\|x_{2}\right\|^{2}}{2}\right)$

(by the arithmetic-geometric mean inequality)

$$
=\frac{2^{r}}{4}\left\|f^{2 r}(|X|)+g^{2 r}\left(\left|Y^{*}\right|\right)\right\|^{\frac{1}{2}}\left\|f^{2 r}(|Y|)+g^{2 r}\left(\left|X^{*}\right|\right)\right\|^{\frac{1}{2}}
$$


Hence, we get the first inequality. Now, applying this fact

$$
\begin{aligned}
& |\langle T \mathbf{x}, \mathbf{x}\rangle|^{r} \\
& =\left|\left\langle X x_{2}, x_{1}\right\rangle+\left\langle Y x_{1}, x_{2}\right\rangle\right|^{r} \\
& \leq\left(\left|\left\langle X x_{2}, x_{1}\right\rangle\right|+\left|\left\langle Y x_{1}, x_{2}\right\rangle\right|\right)^{r} \quad \text { (by the triangular inequality) } \\
& \left.\leq \frac{2^{r}}{2}\left(\left|\left\langle X x_{2}, x_{1}\right\rangle\right|^{r}+\left|\left\langle Y x_{1}, x_{2}\right\rangle\right|^{r}\right) \quad \text { (by the convexity } f(t)=t^{r}\right) \\
& \leq \frac{2^{r}}{2}\left(\left(\left\langle f^{2}(|X|) x_{2}, x_{2}\right\rangle^{\frac{1}{2}}\left\langle g^{2}\left(\left|X^{*}\right|\right) x_{1}, x_{1}\right\rangle^{\frac{1}{2}}\right)^{r}\right. \\
& \left.\quad+\left(\left\langle g^{2}(|Y|) x_{1}, x_{1}\right\rangle^{\frac{1}{2}}\left\langle f^{2}\left(\left|Y^{*}\right|\right) x_{2}, x_{2}\right\rangle^{\frac{1}{2}}\right)^{r}\right) \quad \text { (by Lemma 2.3) }
\end{aligned}
$$

and a similar argument to the proof of the first inequality we have the second inequality and this completes the proof of the theorem.

Theorem 2.4 includes a special case as follows.

Corollary 2.5. Let $T=\left[\begin{array}{cc}0 & X \\ Y & 0\end{array}\right] \in \mathbb{B}\left(\mathscr{H}_{1} \oplus \mathscr{H}_{2}\right), 0 \leq p \leq 1$ and $r \geq 1$. Then

$$
\omega^{r}(T) \leq 2^{r-2}\left\||X|^{2 r p}+\left|Y^{*}\right|^{2 r(1-p)}\right\|^{\frac{1}{2}}\left\||Y|^{2 r p}+\left|X^{*}\right|^{2 r(1-p)}\right\|^{\frac{1}{2}}
$$

and

$$
\omega^{r}(T) \leq 2^{r-2}\left\||X|^{2 r p}+\left|Y^{*}\right|^{2 r p}\right\|^{\frac{1}{2}}\left\||Y|^{2 r(1-p)}+\left|X^{*}\right|^{2 r(1-p)}\right\|^{\frac{1}{2}} .
$$

Proof. The result follows immediately from Theorem 2.4 for $f(t)=t^{p}$ and $g(t)=$ $t^{1-p}(0 \leq p \leq 1)$.

Remark 2.6. Taking $f(t)=g(t)=t^{\frac{1}{2}}(t \in[0, \infty))$ and $r=1$ in Theorem 2.4, we get (see [2, Theorem 4])

$$
\omega(T) \leq \frac{1}{2}\left\||X|+\left.\left|Y^{*}\right|\right|^{\frac{1}{2}}\right\||Y|+\left|X^{*}\right| \|^{\frac{1}{2}},
$$

where $T=\left[\begin{array}{cc}0 & X \\ Y & 0\end{array}\right] \in \mathbb{B}\left(\mathscr{H}_{1} \oplus \mathscr{H}_{2}\right)$.

If we put $Y=X$ in Theorem 2.4, then by using Lemma 2.1(b) we get an extension of Inequality (1.2). 
Corollary 2.7. Let $X \in \mathbb{B}(\mathscr{H}), r \geq 1$ and $f, g$ be nonnegative continuous functions on $[0, \infty)$ satisfying the relation $f(t) g(t)=t(t \in[0, \infty))$. Then

$$
\omega^{r}(X) \leq 2^{r-2}\left\|f^{2 r}(|X|)+g^{2 r}\left(\left|X^{*}\right|\right)\right\|
$$

and

$$
\omega^{r}(X) \leq 2^{r-2}\left\|f^{2 r}(|X|)+f^{2 r}\left(\left|X^{*}\right|\right)\right\|^{\frac{1}{2}}\left\|g^{2 r}(|X|)+g^{2 r}\left(\left|X^{*}\right|\right)\right\|^{\frac{1}{2}}
$$

Corollary 2.8. Let $X, Y \in \mathbb{B}(\mathscr{H})$ and $0 \leq p \leq 1$. Then

$$
\omega^{\frac{r}{2}}(X Y) \leq 2^{r-2}\left\||X|^{2 r p}+\left|Y^{*}\right|^{2 r(1-p)}\right\|^{\frac{1}{2}}\left\||Y|^{2 r p}+\left|X^{*}\right|^{2 r(1-p)}\right\|^{\frac{1}{2}}
$$

and

$$
\omega^{\frac{r}{2}}(X Y) \leq 2^{r-2}\left\||X|^{2 r p}+\left|Y^{*}\right|^{2 r p}\right\|^{\frac{1}{2}}\left\||Y|^{2 r(1-p)}+\left|X^{*}\right|^{2 r(1-p)}\right\|^{\frac{1}{2}}
$$

for $r \geq 1$.

Proof. It follows from the power inequality $\omega^{\frac{1}{2}}\left(T^{2}\right) \leq \omega(T)$ that

$$
\omega^{\frac{1}{2}}\left(T^{2}\right)=\omega^{\frac{1}{2}}\left(\left[\begin{array}{cc}
X Y & 0 \\
0 & Y X
\end{array}\right]\right)=\max \left\{\omega^{\frac{1}{2}}(X Y), \omega^{\frac{1}{2}}(Y X)\right\} .
$$

The required result follows from Corollary 2.5.

Corollary 2.9. Let $X, Y \in \mathbb{B}(\mathscr{H})$ and $r \geq 1$. Then

$$
\left\|X \pm Y^{*}\right\|^{r} \leq 2^{2 r-2}\left\||X|^{r}+\left|Y^{*}\right|^{r}\right\|^{\frac{1}{2}}\left\||Y|^{r}+\left|X^{*}\right|^{r}\right\|^{\frac{1}{2}} .
$$

In particular, if $X$ and $Y$ are normal operators, then

$$
\|X \pm Y\|^{r} \leq 2^{2 r-2}\left\||X|^{r}+|Y|^{r}\right\|
$$

Proof. Applying Lemma 2.1(a) and Corollary 2.5 (for $p=\frac{1}{2}$ ), we have

$$
\begin{aligned}
\left\|X+Y^{*}\right\|^{r} & =\left\|T+T^{*}\right\|^{r} \\
& \leq 2^{r} \max _{\theta \in \mathbb{R}}\left\|\operatorname{Re}\left(e^{i \theta} T\right)\right\|^{r} \\
& =2^{r} \omega^{r}(T) \\
& \leq 2^{2 r-2}\left\||X|^{r}+\left|Y^{*}\right|^{r}\right\|^{\frac{1}{2}}\left\||Y|^{r}+\left|X^{*}\right|^{r}\right\|^{\frac{1}{2}}
\end{aligned}
$$


where $T=\left[\begin{array}{cc}0 & X \\ Y & 0\end{array}\right]$. Similarly,

$$
\begin{aligned}
\left\|X-Y^{*}\right\|^{r} & =\left\|T-T^{*}\right\|^{r} \\
& \leq 2^{r} \max _{\theta \in \mathbb{R}}\left\|\operatorname{Im}\left(e^{i \theta} T\right)\right\|^{r} \\
& =2^{r} \omega^{r}(T) \\
& \leq 2^{2 r-2}\left\||X|^{r}+\left|Y^{*}\right|^{r}\right\|^{\frac{1}{2}}\left\||Y|^{r}+\left|X^{*}\right|^{r}\right\|^{\frac{1}{2}}
\end{aligned}
$$

Hence we get the desired result. For the particular case, observe that $\left|Y^{*}\right|=|Y|$ and $\left|X^{*}\right|=|X|$.

Remark 2.10. It should be mentioned here that inequality (2.2), which has been given earlier, is a generalized form of the well-known inequality (see [4]): if $A$ and $B$ are normal operators, then

$$
\|X+Y\| \leq\||X|+|Y|\|
$$

The normality of $X$ and $Y$ are necessary that means Inequality (2.3) is not true for arbitrary operators $X$ and $Y$; see [12]

In the next theorem, we show another upper bound for numerical radius involving off-diagonal operator matrices.

Theorem 2.11. Let $T=\left[\begin{array}{cc}0 & X \\ Y & 0\end{array}\right] \in \mathbb{B}\left(\mathscr{H}_{1} \oplus \mathscr{H}_{2}\right), r \geq 1$ and $f, g$ be nonnegative continuous functions on $[0, \infty)$ satisfying the relation $f(t) g(t)=t(t \in[0, \infty))$. Then

$$
\omega^{2 r}(T) \leq 4^{r-2}\left(\frac{\left\|\left(f^{2 r}(|X|)+g^{2 r}\left(\left|Y^{*}\right|\right)\right)^{p}\right\|}{p^{2}}+\frac{\left\|\left(f^{2 r}(|Y|)+g^{2 r}\left(\left|X^{*}\right|\right)\right)^{q}\right\|}{q^{2}}\right)
$$

and

$$
\omega^{2 r}(T) \leq 4^{r-2}\left(\frac{\left\|\left(f^{2 r}(|X|)+f^{2 r}\left(\left|Y^{*}\right|\right)\right)^{p}\right\|}{p^{2}}+\frac{\left(\| g^{2 r}(|Y|)+g^{2 r}\left(\left|X^{*}\right|\right)\right)^{q} \|}{q^{2}}\right),
$$

where $\frac{1}{p}+\frac{1}{q}=1$ and $p \geq 1$. 
Proof. If $\mathbf{x}=\left[\begin{array}{c}x_{1} \\ x_{2}\end{array}\right] \in \mathscr{H}_{1} \oplus \mathscr{H}_{2}$ is a unit vector, then by a similar argument to the proof of Theorem 2.4 we have

$$
\begin{aligned}
& |\langle T \mathbf{x}, \mathbf{x}\rangle|^{r} \\
& =\left|\left\langle X x_{2}, x_{1}\right\rangle+\left\langle Y x_{1}, x_{2}\right\rangle\right|^{r} \\
& \leq\left(\left|\left\langle X x_{2}, x_{1}\right\rangle\right|+\left|\left\langle Y x_{1}, x_{2}\right\rangle\right|\right)^{r} \quad \text { (by the triangular inequality) } \\
& \left.\leq \frac{2^{r}}{2}\left(\left|\left\langle X x_{2}, x_{1}\right\rangle\right|^{r}+\left|\left\langle Y x_{1}, x_{2}\right\rangle\right|^{r}\right) \quad \text { (by the convexity } f(t)=t^{r}\right) \\
& \leq \frac{2^{r}}{2}\left(\left(\left\langle f^{2}(|X|) x_{2}, x_{2}\right\rangle^{\frac{1}{2}}\left\langle g^{2}\left(\left|X^{*}\right|\right) x_{1}, x_{1}\right\rangle^{\frac{1}{2}}\right)^{r} \quad(\text { by Lemma } 2.3)\right. \\
& \left.\quad+\left(\left\langle f^{2}(|Y|) x_{1}, x_{1}\right\rangle^{\frac{1}{2}}\left\langle g^{2}\left(\left|Y^{*}\right|\right) x_{2}, x_{2}\right\rangle^{\frac{1}{2}}\right)^{r}\right) \quad \quad\left(\left\langle f^{2}\right)\right. \\
& \leq \frac{2^{r}}{2}\left(\left\langle f^{2 r}(|X|) x_{2}, x_{2}\right\rangle^{\frac{1}{2}}\left\langle g^{2 r}\left|X^{*}\right| x_{1}, x_{1}\right\rangle^{\frac{1}{2}}+\left\langle f^{2 r}(|Y|) x_{1}, x_{1}\right\rangle^{\frac{1}{2}}\left\langle g^{2 r}\left(\left|Y^{*}\right|\right) x_{2}, x_{2}\right\rangle^{\frac{1}{2}}\right)
\end{aligned}
$$

(by Lemma 2.2(a))

$\leq \frac{2^{r}}{2}\left(\left\langle f^{2 r}(|X|) x_{2}, x_{2}\right\rangle+\left\langle g^{2 r}\left(\left|Y^{*}\right|\right) x_{2}, x_{2}\right\rangle\right)^{\frac{1}{2}}$

$\times\left(\left\langle f^{2 r}(|Y|) x_{1}, x_{1}\right\rangle+\left\langle g^{2 r}\left(\left|X^{*}\right|\right) x_{1}, x_{1}\right\rangle\right)^{\frac{1}{2}}$ (by the Cauchy-Schwarz inequality)

$=\frac{2^{r}}{2}\left\langle\left(f^{2 r}(|X|)+g^{2 r}\left(\left|Y^{*}\right|\right)\right) x_{2}, x_{2}\right\rangle^{\frac{1}{2}}\left\langle\left(f^{2 r}(|Y|)+g^{2 r}\left(\left|X^{*}\right|\right)\right) x_{1}, x_{1}\right\rangle^{\frac{1}{2}}$

$\leq \frac{2^{r}}{2}\left(\frac{\left\langle\left(f^{2 r}(|X|)+g^{2 r}\left(\left|Y^{*}\right|\right)\right) x_{2}, x_{2}\right\rangle^{\frac{p}{2}}}{p}+\frac{\left\langle\left(f^{2 r}(|Y|)+g^{2 r}\left(\left|X^{*}\right|\right)\right) x_{1}, x_{1}\right\rangle^{\frac{q}{2}}}{q}\right)$

(by the Young inequality)

$$
\leq \frac{2^{r}}{2}\left(\frac{\left\langle\left(f^{2 r}(|X|)+g^{2 r}\left(\left|Y^{*}\right|\right)\right)^{p} x_{2}, x_{2}\right\rangle^{\frac{1}{2}}}{p}+\frac{\left\langle\left(f^{2 r}(|Y|)+g^{2 r}\left(\left|X^{*}\right|\right)\right)^{q} x_{1}, x_{1}\right\rangle^{\frac{1}{2}}}{q}\right)
$$

(by Lemma 2.2(a))

$\leq \frac{2^{r}}{2}\left(\frac{\left\|\left(f^{2 r}(|X|)+g^{2 r}\left(\left|Y^{*}\right|\right)\right)^{p}\right\|^{\frac{1}{2}}}{p}\left\|x_{2}\right\|+\frac{\left\|\left(f^{2 r}(|Y|)+g^{2 r}\left(\left|X^{*}\right|\right)\right)^{q}\right\|^{\frac{1}{2}}}{q}\left\|x_{1}\right\|\right)$.

Let $\alpha=\frac{\left\|\left(f^{2 r}(|X|)+g^{2 r}\left(\left|Y^{*}\right|\right)\right)^{p}\right\|^{\frac{1}{2}}}{p}$ and $\beta=\frac{\left\|\left(f^{2 r}(|Y|)+g^{2 r}\left(\left|X^{*}\right|\right)\right)^{q}\right\|^{\frac{1}{2}}}{q}$. It follows from

$$
\max _{\left\|x_{1}\right\|^{2}+\left\|x_{2}\right\|^{2}=1}\left(\alpha\left\|x_{1}\right\|+\beta\left\|x_{2}\right\|\right)=\max _{\theta \in[0,2 \pi]}(\alpha \sin \theta+\beta \cos \theta)=\sqrt{\alpha^{2}+\beta^{2}}
$$


and Inequality (2.4) that we deduce

$$
\begin{aligned}
& |\langle T \mathbf{x}, \mathbf{x}\rangle|^{r} \\
& \leq \frac{2^{r}}{2}\left(\frac{\left\|\left(f^{2 r}(|X|)+g^{2 r}\left(\left|Y^{*}\right|\right)\right)^{p}\right\|}{p^{2}}+\frac{\left\|\left(f^{2 r}(|Y|)+g^{2 r}\left(\left|X^{*}\right|\right)\right)^{q}\right\|}{q^{2}}\right)^{\frac{1}{2}} .
\end{aligned}
$$

Taking the supremum over all unit vectors $\mathrm{x} \in \mathscr{H}_{1} \oplus \mathscr{H}_{2}$ we get the first inequality. Now, according to inequality (2.1) and the same argument in the proof of the first inequality, we obtain the second inequality.

Remark 2.12. If $T=\left[\begin{array}{cc}0 & X \\ Y & 0\end{array}\right] \in \mathbb{B}\left(\mathscr{H}_{1} \oplus \mathscr{H}_{2}\right)$ and $\frac{1}{p}+\frac{1}{q}=1$, then by using Theorem 2.4 and the Young inequality we obtain the inequalities

$$
\omega^{r}(T) \leq 2^{r-2}\left(\frac{\left\|f^{2 r}(|X|)+g^{2 r}\left(\left|Y^{*}\right|\right)\right\|^{\frac{p}{2}}}{p}+\frac{\left\|f^{2 r}(|Y|)+g^{2 r}\left(\left|X^{*}\right|\right)\right\|^{\frac{q}{2}}}{q}\right)
$$

and

$$
\omega^{r}(T) \leq 2^{r-2}\left(\frac{\left\|f^{2 r}(|X|)+f^{2 r}\left(\left|Y^{*}\right|\right)\right\|^{\frac{p}{2}}}{p}+\frac{\left\|g^{2 r}(|Y|)+g^{2 r}\left(\left|X^{*}\right|\right)\right\|^{\frac{q}{2}}}{q}\right)
$$

where $r \geq 1$ and $f, g$ are nonnegative continuous functions on $[0, \infty)$ satisfying the relation $f(t) g(t)=t(t \in[0, \infty))$. Now, Theorem 2.11 shows some other upper bounds for $\omega(T)$.

In the special case of Theorem 2.11 for $Y=X$ and $p=q=2$, we have the next result.

Corollary 2.13. Let $X \in \mathbb{B}(\mathscr{H}), r \geq 1$ and $f, g$ be nonnegative continuous functions on $[0, \infty)$ satisfying the relation $f(t) g(t)=t(t \in[0, \infty))$. Then

$$
\omega^{2 r}(X) \leq 2^{2 r-3}\left\|\left(f^{2 r}(|X|)+g^{2 r}\left(\left|X^{*}\right|\right)\right)^{2}\right\|
$$

and

$$
\omega^{2 r}(T) \leq 2^{2 r-4}\left(\left\|\left(f^{2 r}(|X|)+f^{2 r}\left(\left|X^{*}\right|\right)\right)^{2}\right\|+\left(\| g^{2 r}(|X|)+g^{2 r}\left(\left|X^{*}\right|\right)\right)^{2} \|\right) .
$$

Applying Inequality (1.1) we obtain the following theorem. 
Theorem 2.14. Let $T=\left[\begin{array}{cc}0 & X \\ Y & 0\end{array}\right] \in \mathbb{B}\left(\mathscr{H}_{1} \oplus \mathscr{H}_{2}\right)$ and $f, g$ be nonnegative continuous functions on $[0, \infty)$ satisfying the relation $f(t) g(t)=t(t \in[0, \infty))$. Then for $r \geq 1$

$$
\omega^{r}(T) \leq 2^{r-2}\left(\left\|f^{2 r}(|X|)+g^{2 r}\left(\left|Y^{*}\right|\right)\right\|+\left\|f^{2 r}(|Y|)+g^{2 r}\left(\left|X^{*}\right|\right)\right\|\right)-2^{r-2} \inf _{\left\|\left(x_{1}, x_{2}\right)\right\|=1} \zeta\left(x_{1}, x_{2}\right),
$$

where

$$
\zeta\left(x_{1}, x_{2}\right)=\left(\left\langle\left(f^{2 r}(|X|)+g^{2 r}\left(\left|Y^{*}\right|\right)\right) x_{2}, x_{2}\right\rangle^{\frac{1}{2}}-\left\langle\left(f^{2 r}(|Y|)+g^{2 r}\left(\left|X^{*}\right|\right)\right) x_{1}, x_{1}\right\rangle^{\frac{1}{2}}\right)^{2} .
$$

Proof. Let $\mathbf{x}=\left[\begin{array}{l}x_{1} \\ x_{2}\end{array}\right] \in \mathscr{H}_{1} \oplus \mathscr{H}_{2}$ be a unit vector. Then

$$
\begin{aligned}
|\langle T \mathbf{x}, \mathbf{x}\rangle|^{r} \\
\quad=\left|\left\langle X x_{2}, x_{1}\right\rangle+\left\langle Y x_{1}, x_{2}\right\rangle\right|^{r} \\
\leq\left(\left|\left\langle X x_{2}, x_{1}\right\rangle\right|+\left|\left\langle Y x_{1}, x_{2}\right\rangle\right|\right)^{r} \quad \text { (by the triangular inequality) } \\
\left.\leq \frac{2^{r}}{2}\left(\left|\left\langle X x_{2}, x_{1}\right\rangle\right|^{r}+\left|\left\langle Y x_{1}, x_{2}\right\rangle\right|^{r}\right) \quad \text { (by the convexity } f(t)=t^{r}\right) \\
\leq \frac{2^{r}}{2}\left(\left\langle f^{2}(|X|) x_{2}, x_{2}\right\rangle^{\frac{r}{2}}\left\langle g^{2}\left(\left|X^{*}\right|\right) x_{1}, x_{1}\right\rangle^{\frac{r}{2}}+\left\langle f^{2}(|Y|) x_{1}, x_{1}\right\rangle^{\frac{r}{2}}\left\langle f^{2}\left(\left|Y^{*}\right|\right) x_{2}, x_{2}\right\rangle^{\frac{r}{2}}\right)
\end{aligned}
$$

(by Lemma 2.3)

$$
\begin{aligned}
\leq & \frac{2^{r}}{2}\left(\left\langle f^{2 r}(|X|) x_{2}, x_{2}\right\rangle^{\frac{1}{2}}\left\langle g^{2 r}\left(\left|X^{*}\right|\right) x_{1}, x_{1}\right\rangle^{\frac{1}{2}}+\left\langle f^{2 r}(|Y|) x_{1}, x_{1}\right\rangle^{\frac{1}{2}}\left\langle g^{2 r}\left(\left|Y^{*}\right|\right) x_{2}, x_{2}\right\rangle^{\frac{1}{2}}\right) \\
\leq & \left.\frac{2^{r}}{2}\left(\left\langle f^{2 r}(|X|) x_{2}, x_{2}\right\rangle+\left\langle g^{2 r}\left(\left|Y^{*}\right|\right) x_{2}, x_{2}\right\rangle\right)^{\frac{1}{2}}\left(f^{2 r}(|Y|) x_{1}, x_{1}\right\rangle+\left\langle g^{2 r}\left(\left|X^{*}\right|\right) x_{1}, x_{1}\right\rangle\right)^{\frac{1}{2}} \\
= & \frac{2^{r}}{2}\left\langle\left(f^{2 r}(|X|)+g^{2 r}\left(\left|Y^{*}\right|\right)\right) x_{2}, x_{2}\right\rangle^{\frac{1}{2}}\left\langle\left(f^{2 r}(|Y|)+g^{2 r}\left(\left|X^{*}\right|\right)\right) x_{1}, x_{1}\right\rangle^{\frac{1}{2}} \\
\leq & \frac{2^{r}}{4}\left(\left\langle\left(f^{2 r}(|X|)+g^{2 r}\left(\left|Y^{*}\right|\right)\right) x_{2}, x_{2}\right\rangle+\left\langle\left(f^{2 r}(|Y|)+g^{2 r}\left(\left|X^{*}\right|\right)\right) x_{1}, x_{1}\right\rangle\right) \\
& -\frac{2^{r}}{4}\left(\left\langle\left(f^{2 r}(|X|)+g^{2 r}\left(\left|Y^{*}\right|\right)\right) x_{2}, x_{2}\right\rangle^{\frac{1}{2}}-\left\langle\left(f^{2 r}(|Y|)+g^{2 r}\left(\left|X^{*}\right|\right)\right) x_{1}, x_{1}\right\rangle^{\frac{1}{2}}\right)^{2}
\end{aligned}
$$

(by Inequality (1.1))

$$
\begin{aligned}
\leq & \frac{2^{r}}{4}\left(\left\|f^{2 r}(|X|)+g^{2 r}\left(\left|Y^{*}\right|\right)\right\|+\left\|f^{2 r}(|Y|)+g^{2 r}\left(\left|X^{*}\right|\right)\right\|\right) \\
& -\frac{2^{r}}{4}\left(\left\langle\left(f^{2 r}(|X|)+g^{2 r}\left(\left|Y^{*}\right|\right)\right) x_{2}, x_{2}\right\rangle^{\frac{1}{2}}-\left\langle\left(f^{2 r}(|Y|)+g^{2 r}\left(\left|X^{*}\right|\right)\right) x_{1}, x_{1}\right\rangle^{\frac{1}{2}}\right)^{2} .
\end{aligned}
$$

Taking the supremum over all unit vectors $\mathbf{x}=\left[\begin{array}{l}x_{1} \\ x_{2}\end{array}\right] \in \mathscr{H}_{1} \oplus \mathscr{H}_{2}$ we get the desired inequality. 
If we put $Y=X$ in Theorem 2.14, then we get next result.

Corollary 2.15. Let $X \in \mathbb{B}(\mathscr{H})$ and $f, g$ be nonnegative continuous functions on $[0, \infty)$ satisfying the relation $f(t) g(t)=t(t \in[0, \infty))$. Then for $r \geq 1$

$$
\omega^{r}(X) \leq 2^{r-1}\left\|f^{2 r}(|X|)+g^{2 r}\left(\left|X^{*}\right|\right)\right\|-2^{r-2} \inf _{\left\|\left(x_{1}, x_{2}\right)\right\|=1} \zeta\left(x_{1}, x_{2}\right),
$$

where

$$
\zeta\left(x_{1}, x_{2}\right)=\left(\left\langle\left(f^{2 r}(|X|)+g^{2 r}\left(\left|X^{*}\right|\right)\right) x_{2}, x_{2}\right\rangle^{\frac{1}{2}}-\left\langle\left(f^{2 r}(|X|)+g^{2 r}\left(\left|X^{*}\right|\right)\right) x_{1}, x_{1}\right\rangle^{\frac{1}{2}}\right)^{2} .
$$

Remark 2.16. If $\mathbf{x}=\left[\begin{array}{l}x_{1} \\ x_{2}\end{array}\right] \in \mathscr{H}_{1} \oplus \mathscr{H}_{2}$ is a unit vector, then by using the inequality

$$
\begin{aligned}
|\langle T \mathbf{x}, \mathbf{x}\rangle|^{r} \\
\quad=\left|\left\langle X x_{2}, x_{1}\right\rangle+\left\langle Y x_{1}, x_{2}\right\rangle\right|^{r} \\
\leq\left(\left|\left\langle X x_{2}, x_{1}\right\rangle\right|+\left|\left\langle Y x_{1}, x_{2}\right\rangle\right|\right)^{r} \\
\quad \leq \frac{2^{r}}{2}\left(\left|\left\langle X x_{2}, x_{1}\right\rangle\right|^{r}+\left|\left\langle Y x_{1}, x_{2}\right\rangle\right|^{r}\right) \\
\quad \leq \frac{2^{r}}{2}\left(\left\langle f^{2}(|X|) x_{2}, x_{2}\right\rangle^{\frac{r}{2}}\left\langle g^{2}\left(\left|X^{*}\right|\right) x_{1}, x_{1}\right\rangle^{\frac{r}{2}}\left\langle g^{2}(|X|) x_{2}, x_{2}\right\rangle^{\frac{r}{2}}\left\langle f^{2}\left(\left|X^{*}\right|\right) x_{1}, x_{1}\right\rangle^{\frac{r}{2}}\right)
\end{aligned}
$$

and the same argument in the proof if Theorem 2.14 we get the following inequality

$$
\omega^{r}(T) \leq \frac{2^{r}}{4}\left(\left\|f^{2 r}(|X|)+f^{2 r}\left(\left|Y^{*}\right|\right)\right\|+\left\|g^{2 r}(|Y|)+g^{2 r}\left(\left|X^{*}\right|\right)\right\|\right)-\frac{2^{r}}{4} \inf _{\left\|\left(x_{1}, x_{2}\right)\right\|=1} \zeta\left(x_{1}, x_{2}\right),
$$

where $T=\left[\begin{array}{cc}0 & X \\ Y & 0\end{array}\right] \in \mathbb{B}\left(\mathscr{H}_{1} \oplus \mathscr{H}_{2}\right), f, g$ are nonnegative continuous functions on $[0, \infty)$ satisfying the relation $f(t) g(t)=t(t \in[0, \infty)), r \geq 1$ and

$$
\zeta\left(x_{1}, x_{2}\right)=\left(\left\langle\left(f^{2 r}(|X|)+f^{2 r}\left(\left|Y^{*}\right|\right)\right) x_{2}, x_{2}\right\rangle^{\frac{1}{2}}-\left\langle\left(g^{2 r}(|Y|)+g^{2 r}\left(\left|X^{*}\right|\right)\right) x_{1}, x_{1}\right\rangle^{\frac{1}{2}}\right)^{2} .
$$

\section{SOME UPPER BOUNDS FOR $\omega_{p}$}

In this section, we obtain some upper bounds for $\omega_{P}$. We first show the following theorem.

Theorem 3.1. Let $\widetilde{S}_{i}=\left[\begin{array}{cc}A_{i} & 0 \\ 0 & B_{i}\end{array}\right], \widetilde{T}_{i}=\left[\begin{array}{cc}0 & X_{i} \\ Y_{i} & 0\end{array}\right]$ and $\widetilde{U}_{i}=\left[\begin{array}{cc}C_{i} & 0 \\ 0 & D_{i}\end{array}\right]$ be operators matrices in $\mathbb{B}\left(\mathscr{H}_{1} \oplus \mathscr{H}_{2}\right)(1 \leq i \leq n)$ such that $A_{i}, B_{i}, C_{i}$ and $D_{i}$ are contractions. 
Then

$$
\begin{aligned}
& \omega_{p}^{p}\left(\widetilde{S}_{1}^{*} \widetilde{T}_{1} \widetilde{U}_{1}, \cdots, \widetilde{S}_{n}^{*} \widetilde{T}_{n} \widetilde{U}_{n}\right) \\
& \quad \leq 2^{p-2} \sum_{i=1}^{n}\left\|D_{i}^{*} f^{2 p}\left(\left|X_{i}\right|\right) D_{i}+B_{i}^{*} g^{2 p}\left(\left|Y_{i}^{*}\right|\right) B_{i}\right\|^{\frac{1}{2}}\left\|C_{i}^{*} f^{2 p}\left(\left|Y_{i}\right|\right) C_{i}+A_{i}^{*} g^{2 p}\left(\left|X_{i}^{*}\right|\right) A_{i}\right\|^{\frac{1}{2}}
\end{aligned}
$$

and

$$
\begin{aligned}
& \omega_{p}^{p}\left(\widetilde{S}_{1}^{*} \widetilde{T}_{1} \widetilde{U}_{1}, \cdots, \widetilde{S}_{n}^{*} \widetilde{T}_{n} \widetilde{U}_{n}\right) \\
& \quad \leq 2^{p-2} \sum_{i=1}^{n}\left\|D_{i}^{*} f^{2 p}\left(\left|X_{i}\right|\right) D_{i}+B_{i}^{*} f^{2 p}\left(\left|Y_{i}^{*}\right|\right) B_{i}\right\|^{\frac{1}{2}}\left\|C_{i}^{*} g^{2 p}\left(\left|Y_{i}\right|\right) C_{i}+A_{i}^{*} g^{2 p}\left(\left|X_{i}^{*}\right|\right) A_{i}\right\|^{\frac{1}{2}},
\end{aligned}
$$

where $p \geq 1$.

Proof. For any unit vector $\mathbf{x}=\left[\begin{array}{l}x_{1} \\ x_{2}\end{array}\right] \in \mathscr{H}_{1} \oplus \mathscr{H}_{2}$ we have

$$
\begin{aligned}
\sum_{i=1}^{n}\left|\left\langle T_{i} \mathbf{x}, \mathbf{x}\right\rangle\right|^{p} & \sum_{i=1}^{n}\left|\left\langle A_{i}^{*} X_{i} D_{i} x_{2}, x_{1}\right\rangle+\left\langle B_{i}^{*} Y_{i} C_{i} x_{1}, x_{2}\right\rangle\right|^{p} \\
\leq & \sum_{i=1}^{n}\left(\left|\left\langle A_{i}^{*} X_{i} D_{i} x_{2}, x_{1}\right\rangle\right|+\left|\left\langle B_{i}^{*} Y_{i} C_{i} x_{1}, x_{2}\right\rangle\right|\right)^{p} \quad \text { (by the triangular inequality) } \\
\leq & \left.\frac{2^{p}}{2} \sum_{i=1}^{n}\left|\left\langle A_{i}^{*} X_{i} D_{i} x_{2}, x_{1}\right\rangle\right|^{p}+\left|\left\langle B_{i}^{*} Y_{i} C_{i} x_{1}, x_{2}\right\rangle\right|^{p} \quad \text { (by the convexity } f(t)=t^{p}\right) \\
= & \frac{2^{p}}{2} \sum_{i=1}^{n}\left|\left\langle X_{i} D_{i} x_{2}, A_{i} x_{1}\right\rangle\right|^{p}+\left|\left\langle Y_{i} C_{i} x_{1}, B_{i} x_{2}\right\rangle\right|^{p} \\
\leq & \frac{2^{p}}{2} \sum_{i=1}^{n}\left\langle f^{2}\left(\left|X_{i}\right|\right) D_{i} x_{2}, D_{i} x_{2}\right\rangle^{\frac{p}{2}}\left\langle g^{2}\left(\left|X_{i}^{*}\right|\right) A_{i} x_{1}, A_{i} x_{1}\right\rangle^{\frac{p}{2}} \\
& +\left\langle f^{2}\left(\left|Y_{i}\right|\right) C_{i} x_{1}, C_{i} x_{1}\right\rangle^{\frac{p}{2}}\left\langle g^{2}\left(\left|Y_{i}^{*}\right|\right) B_{i} x_{2}, B_{i} x_{2}\right\rangle^{\frac{p}{2}} \quad \text { (by Lemma 2.3) } \\
\leq & \frac{2^{p}}{2} \sum_{i=1}^{n}\left\langle f^{2 p}\left(\left|X_{i}\right|\right) D_{i} x_{2}, D_{i} x_{2}\right\rangle^{\frac{1}{2}}\left\langle g^{2 p}\left(\left|X_{i}^{*}\right|\right) A_{i} x_{1}, A_{i} x_{1}\right\rangle^{\frac{1}{2}} \\
& +\left\langle f^{2 p}\left(\left|Y_{i}\right|\right) C_{i} x_{1}, C_{i} x_{1}\right\rangle^{\frac{1}{2}}\left\langle g^{2 p}\left(\left|Y_{i}^{*}\right|\right) B_{i} x_{2}, B_{i} x_{2}\right\rangle^{\frac{1}{2}} \quad \quad \text { (by Lemma 2.2(a)) } \\
= & \frac{2^{p}}{2} \sum_{i=1}^{n}\left\langle D_{i}^{*} f^{2 p}\left(\left|X_{i}\right|\right) D_{i} x_{2}, x_{2}\right\rangle^{\frac{1}{2}}\left\langle A_{i}^{*} g^{2 p}\left(\left|X_{i}^{*}\right|\right) A_{i} x_{1}, x_{1}\right\rangle^{\frac{1}{2}} \\
& +\left\langle C_{i}^{*} f^{2 p}\left(\left|Y_{i}\right|\right) C_{i} x_{1}, x_{1}\right\rangle^{\frac{1}{2}}\left\langle B_{i}^{*} g^{2 p}\left(\left|Y_{i}^{*}\right|\right) B_{i} x_{2}, x_{2}\right\rangle^{\frac{1}{2}} \quad \\
\leq & \frac{2^{p}}{2} \sum_{i=1}^{n}\left(\left\langle D_{i}^{*} f^{2 p}\left(\left|X_{i}\right|\right) D_{i} x_{2}, x_{2}\right\rangle+\left\langle B_{i}^{*} g^{2 p}\left(\left|Y_{i}^{*}\right|\right) B_{i} x_{2}, x_{2}\right\rangle\right)^{\frac{1}{2}} \\
& \times\left(\left\langle C_{i}^{*} f^{2 p}\left(\left|Y_{i}\right|\right) C_{i} x_{1}, x_{1}\right\rangle+\left\langle A_{i}^{*} g^{2 p}\left(\left|X_{i}^{*}\right|\right) A_{i} x_{1}, x_{1}\right\rangle\right)^{\frac{1}{2}} \quad \text { (by the Cauchy-Schwarz inequality)}
\end{aligned}
$$




$$
\begin{aligned}
= & \frac{2^{p}}{2} \sum_{i=1}^{n}\left(\left\langle\left(D_{i}^{*} f^{2 p}\left(\left|X_{i}\right|\right) D_{i}+B_{i}^{*} g^{2 p}\left(\left|Y_{i}^{*}\right|\right) B_{i}\right) x_{2}, x_{2}\right\rangle\right)^{\frac{1}{2}} \\
& \times\left(\left\langle\left(C_{i}^{*} f^{2 p}\left(\left|Y_{i}\right|\right) C_{i}+A_{i}^{*} g^{2 p}\left(\left|X_{i}^{*}\right|\right) A_{i}\right) x_{1}, x_{1}\right\rangle\right)^{\frac{1}{2}} \\
\leq & \frac{2^{p}}{2} \sum_{i=1}^{n}\left\|D_{i}^{*} f^{2 p}\left(\left|X_{i}\right|\right) D_{i}+B_{i}^{*} g^{2 p}\left(\left|Y_{i}^{*}\right|\right) B_{i}\right\|^{\frac{1}{2}}\left\|C_{i}^{*} f^{2 p}\left(\left|Y_{i}\right|\right) C_{i}+A_{i}^{*} g^{2 p}\left(\left|X_{i}^{*}\right|\right) A_{i}\right\|^{\frac{1}{2}}\left\|x_{1}\right\|\left\|x_{2}\right\| \\
= & \frac{2^{p}}{2} \sum_{i=1}^{n}\left\|D_{i}^{*} f^{2 p}\left(\left|X_{i}\right|\right) D_{i}+B_{i}^{*} g^{2 p}\left(\left|Y_{i}^{*}\right|\right) B_{i}\right\|^{\frac{1}{2}} \\
& \times\left\|C_{i}^{*} f^{2 p}\left(\left|Y_{i}\right|\right) C_{i}+A_{i}^{*} g^{2 p}\left(\left|X_{i}^{*}\right|\right) A_{i}\right\|^{\frac{1}{2}}\left(\frac{\left\|x_{1}\right\|^{2}+\left\|x_{2}\right\|^{2}}{2}\right) \\
= & \frac{2^{p}}{4} \sum_{i=1}^{n}\left\|D_{i}^{*} f^{2 p}\left(\left|X_{i}\right|\right) D_{i}+B_{i}^{*} g^{2 p}\left(\left|Y_{i}^{*}\right|\right) B_{i}\right\|^{\frac{1}{2}}\left\|C_{i}^{*} f^{2 p}\left(\left|Y_{i}\right|\right) C_{i}+A_{i}^{*} g^{2 p}\left(\left|X_{i}^{*}\right|\right) A_{i}\right\|^{\frac{1}{2}} .
\end{aligned}
$$

Taking the supremum over all unit vectors $\mathrm{x} \in \mathscr{H}_{1} \oplus \mathscr{H}_{2}$ we obtain the first inequality. Using the inequality

$$
\begin{aligned}
\sum_{i=1}^{n}\left|\left\langle T_{i} \mathbf{x}, \mathbf{x}\right\rangle\right|^{p} \\
=\sum_{i=1}^{n}\left|\left\langle A_{i}^{*} X_{i} D_{i} x_{2}, x_{1}\right\rangle+\left\langle B_{i}^{*} Y_{i} C_{i} x_{1}, x_{2}\right\rangle\right|^{p} \\
\leq \sum_{i=1}^{n}\left(\left|\left\langle A_{i}^{*} X_{i} D_{i} x_{2}, x_{1}\right\rangle\right|+\left|\left\langle B_{i}^{*} Y_{i} C_{i} x_{1}, x_{2}\right\rangle\right|\right)^{p} \quad \text { (by the triangular inequality) } \\
\left.\leq \frac{2^{p}}{2} \sum_{i=1}^{n}\left|\left\langle A_{i}^{*} X_{i} D_{i} x_{2}, x_{1}\right\rangle\right|^{p}+\left|\left\langle B_{i}^{*} Y_{i} C_{i} x_{1}, x_{2}\right\rangle\right|^{p} \quad \text { (by the convexity } f(t)=t^{p}\right) \\
=\frac{2^{p}}{2} \sum_{i=1}^{n}\left|\left\langle X_{i} D_{i} x_{2}, A_{i} x_{1}\right\rangle\right|^{p}+\left|\left\langle Y_{i} C_{i} x_{1}, B_{i} x_{2}\right\rangle\right|^{p} \quad \\
\leq \frac{2^{p}}{2} \sum_{i=1}^{n}\left\langle f^{2}\left(\left|X_{i}\right|\right) D_{i} x_{2}, D_{i} x_{2}\right\rangle^{\frac{p}{2}}\left\langle g^{2}\left(\left|X_{i}^{*}\right|\right) A_{i} x_{1}, A_{i} x_{1}\right\rangle^{\frac{p}{2}} \\
\quad+\left\langle g^{2}\left(\left|Y_{i}\right|\right) C_{i} x_{1}, C_{i} x_{1}\right\rangle^{\frac{p}{2}}\left\langle f^{2}\left(\left|Y_{i}^{*}\right|\right) B_{i} x_{2}, B_{i} x_{2}\right\rangle^{\frac{p}{2}}
\end{aligned}
$$

(by Lemma 2.3)

and a similar fashion in the proof of the first inequality we reach the second inequality.

In the special case of Theorem 3.1 for $A_{i}=B_{i}=C_{i}=D_{i}=I(1 \leq i \leq n)$ we have the next result. 
Corollary 3.2. Let $T_{i}=\left[\begin{array}{cc}0 & X_{i} \\ Y_{i} & 0\end{array}\right] \in \mathbb{B}\left(\mathscr{H}_{1} \oplus \mathscr{H}_{2}\right)(1 \leq j \leq n)$. Then

$$
\omega_{p}^{p}\left(T_{1}, T_{2}, \cdots, T_{n}\right) \leq 2^{p-2} \sum_{i=1}^{n}\left\|f^{2 p}\left(\left|X_{i}\right|\right)+g^{2 p}\left(\left|Y_{i}^{*}\right|\right)\right\|^{\frac{1}{2}}\left\|f^{2 p}\left(\left|Y_{i}\right|\right)+g^{2 p}\left(\left|X_{i}^{*}\right|\right)\right\|^{\frac{1}{2}}
$$

and

$$
\omega_{p}^{p}\left(T_{1}, T_{2}, \cdots, T_{n}\right) \leq 2^{p-2} \sum_{i=1}^{n}\left\|f^{2 p}\left(\left|X_{i}\right|\right)+f^{2 p}\left(\left|Y_{i}^{*}\right|\right)\right\|^{\frac{1}{2}}\left\|g^{2 p}\left(\left|Y_{i}\right|\right)+g^{2 p}\left(\left|X_{i}^{*}\right|\right)\right\|^{\frac{1}{2}}
$$

for $p \geq 1$.

If we put $f(t)=g(t)=t^{\frac{1}{2}}(t \in[0, \infty))$, then we get the next result.

Corollary 3.3. Let $T_{i}=\left[\begin{array}{cc}0 & X_{i} \\ Y_{i} & 0\end{array}\right] \in \mathbb{B}\left(\mathscr{H}_{1} \oplus \mathscr{H}_{2}\right)(1 \leq j \leq n)$. Then

$$
\omega_{p}^{p}\left(T_{1}, T_{2}, \cdots, T_{n}\right) \leq 2^{p-2} \sum_{i=1}^{n}\left\|\left|X_{i}\right|^{p}+\left|Y_{i}^{*}\right|^{p}\right\|^{\frac{1}{2}}\left\|\left|Y_{i}\right|^{p}+\left|X_{i}^{*}\right|^{p}\right\|^{\frac{1}{2}}
$$

for $p \geq 1$.

Theorem 3.4. Let $T_{i}=\left[\begin{array}{cc}A_{i} & B_{i} \\ C_{i} & D_{i}\end{array}\right] \in \mathbb{B}\left(\mathscr{H}_{1} \oplus \mathscr{H}_{2}\right)(1 \leq i \leq n)$ and $p \geq 1$. Then

$$
\begin{aligned}
& \omega_{p}^{p}\left(T_{1}, \ldots, T_{n}\right) \\
& \quad \leq 2^{-p} \sum_{i=1}^{n}\left(\omega\left(A_{i}\right)+\omega\left(D_{i}\right)+\sqrt{\left(\omega\left(A_{i}\right)-\omega\left(D_{i}\right)\right)^{2}+\left(\left\|B_{i}\right\|+\left\|C_{i}\right\|\right)^{2}}\right)^{p} .
\end{aligned}
$$

In particular,

$$
\omega\left(\left[\begin{array}{ll}
A & B \\
C & D
\end{array}\right]\right) \leq \frac{1}{2}\left(\omega(A)+\omega(D)+\sqrt{(\omega(A)-\omega(D))^{2}+(\|B\|+\|C\|)^{2}}\right) .
$$

Proof. Let $\mathbf{x}=\left[\begin{array}{l}x_{1} \\ x_{2}\end{array}\right]$ be a unit vector in $\mathscr{H}_{1} \oplus \mathscr{H}_{2}$. Then 


$$
\begin{aligned}
\left|\left\langle T_{i} \mathbf{x}, \mathbf{x}\right\rangle\right| & =\left|\left\langle\left[\begin{array}{cc}
A_{i} & B_{i} \\
C_{i} & D_{i}
\end{array}\right]\left[\begin{array}{l}
x_{1} \\
x_{2}
\end{array}\right],\left[\begin{array}{l}
x_{1} \\
x_{2}
\end{array}\right]\right\rangle\right| \\
& =\left|\left\langle\left[\begin{array}{c}
A_{i} x_{1}+B_{i} x_{2} \\
C_{i} x_{1}+D_{i} x_{2}
\end{array}\right],\left[\begin{array}{c}
x_{1} \\
x_{2}
\end{array}\right]\right\rangle\right| \\
& =\left|\left\langle A_{i} x_{1}, x_{1}\right\rangle+\left\langle B_{i} x_{2}, x_{1}\right\rangle+\left\langle C_{i} x_{1}, x_{2}\right\rangle+\left\langle D_{i} x_{2}, x_{2}\right\rangle\right| \\
& \leq\left|\left\langle A_{i} x_{1}, x_{1}\right\rangle\right|+\left|\left\langle B_{i} x_{2}, x_{1}\right\rangle\right|+\left|\left\langle C_{i} x_{1}, x_{2}\right\rangle\right|+\left|\left\langle D_{i} x_{2}, x_{2}\right\rangle\right|
\end{aligned}
$$

Thus,

$$
\begin{aligned}
& \omega_{p}^{p}\left(T_{1}, \ldots, T_{n}\right) \\
& =\sup _{\|\mathbf{x}\|=1} \sum_{i=1}^{n}\left|\left\langle T_{i} \mathbf{x}, \mathbf{x}\right\rangle\right|^{p} \\
& \leq \sup _{\left\|x_{1}\right\|^{2}+\left\|x_{2}\right\|^{2}=1} \sum_{i=1}^{n}\left(\left|\left\langle A_{i} x_{1}, x_{1}\right\rangle\right|+\left|\left\langle B_{i} x_{2}, x_{1}\right\rangle\right|+\left|\left\langle C_{i} x_{1}, x_{2}\right\rangle\right|+\left|\left\langle D_{i} x_{2}, x_{2}\right\rangle\right|\right)^{p} \\
& \leq \sum_{i=1}^{n}\left(\sup _{\left\|x_{1}\right\|^{2}+\|y\|^{2}=1}\left(\left|\left\langle A_{i} x_{1}, x_{1}\right\rangle\right|+\left|\left\langle B_{i} x_{2}, x_{1}\right\rangle\right|+\left|\left\langle C_{i} x_{1}, x_{2}\right\rangle\right|+\left|\left\langle D_{i} x_{2}, x_{2}\right\rangle\right|\right)\right)^{p} \\
& \leq \sum_{i=1}^{n}\left(\sup _{\left\|x_{1}\right\|^{2}+\left\|x_{2}\right\|^{2}=1}\left(\omega\left(A_{i}\right)\left\|x_{1}\right\|^{2}+\omega\left(D_{i}\right)\left\|x_{2}\right\|^{2}+\left(\left\|B_{i}\right\|+\left\|C_{i}\right\|\right)\left\|x_{1}\right\|\left\|x_{2}\right\|\right)\right)^{p} \\
& =\sum_{i=1}^{n}\left(\sup _{\theta \in[0,2 \pi]}\left(\omega\left(A_{i}\right) \cos ^{2} \theta+\omega\left(D_{i}\right) \sin ^{2} \theta+\left(\left\|B_{i}\right\|+\left\|C_{i}\right\|\right) \cos \theta \sin \theta\right)\right)^{p} \\
& =2^{-p} \sum_{i=1}^{n}\left(\omega\left(A_{i}\right)+\omega\left(D_{i}\right)+\sqrt{\left(\omega\left(A_{i}\right)-\omega\left(D_{i}\right)\right)^{2}+\left(\left\|B_{i}\right\|+\left\|C_{i}\right\|\right)^{2}}\right)^{p} .
\end{aligned}
$$

This completes the proof.

For $A_{i}=D_{i}$ and $B_{i}=C_{i}(1 \leq i \leq n)$ we get the following result.

Corollary 3.5. Let $T_{i}=\left[\begin{array}{cc} \pm A_{i} & \pm B_{i} \\ \pm B_{i} & \pm A_{i}\end{array}\right]$ be an operator matrix with $A_{i}, B_{i} \in \mathbb{B}(\mathscr{H})$ $(1 \leq i \leq n)$. Then for all $p \geq 1$,

$$
\omega_{p}^{p}\left(T_{1}, \ldots, T_{n}\right) \leq \sum_{i=1}^{n}\left(\omega\left(A_{i}\right)+\left\|B_{i}\right\|\right)^{p}
$$


In particular, if $A, B \in \mathbb{B}(\mathscr{H})$, then

$$
\omega\left(\left[\begin{array}{cc} 
\pm A & \pm B \\
\pm B & \pm A
\end{array}\right]\right) \leq \omega(A)+\|B\|
$$

If we take $B_{i}=C_{i}=0(1 \leq i \leq n)$ in Theorem 3.4, then we get the following inequality.

Corollary 3.6. Let $T_{i}=\left[\begin{array}{cc}A_{i} & 0 \\ 0 & D_{i}\end{array}\right] \in \mathbb{B}\left(\mathscr{H}_{1} \oplus \mathscr{H}_{2}\right)(1 \leq i \leq n)$. Then for all $p \geq 1$,

$$
\omega_{p}^{p}\left(T_{1}, \ldots, T_{n}\right) \leq \sum_{i=1}^{n} \max \left(\omega^{p}\left(A_{i}\right), \omega^{p}\left(D_{i}\right)\right)
$$

For $C_{i}=D_{i}=0(1 \leq i \leq n)$ we obtain a result that generalize and refine the inequality $\omega\left(\left[\begin{array}{cc}A & B \\ 0 & 0\end{array}\right]\right) \leq \omega(A)+\frac{\|B\|}{2}$.

Corollary 3.7. Let $T_{i}=\left[\begin{array}{cc}A_{i} & B_{i} \\ 0 & 0\end{array}\right] \in \mathbb{B}\left(\mathscr{H}_{1} \oplus \mathscr{H}_{2}\right)(1 \leq i \leq n)$ and $p \geq 1$. Then

$$
\omega_{p}^{p}\left(T_{1}, \ldots, T_{n}\right) \leq 2^{-p} \sum_{i=1}^{n}\left(\omega\left(A_{i}\right)+\sqrt{\omega^{2}\left(A_{i}\right)+\left\|B_{i}\right\|^{2}}\right)^{p} .
$$

In particular,

$$
\omega\left(\left[\begin{array}{cc}
A & B \\
0 & 0
\end{array}\right]\right) \leq \frac{1}{2}\left(\omega(A)+\sqrt{\omega^{2}(A)+\|B\|^{2}}\right) .
$$

If we put $A_{i}=D_{i}=0(1 \leq i \leq n)$, then we deduce

Corollary 3.8. Let $T_{i}=\left[\begin{array}{cc}0 & B_{i} \\ C_{i} & 0\end{array}\right] \in \mathbb{B}\left(\mathscr{H}_{1} \oplus \mathscr{H}_{2}\right)(1 \leq i \leq n)$ and $p \geq 1$. The

$$
\omega_{p}^{p}\left(T_{1}, \ldots, T_{n}\right) \leq 2^{-p} \sum_{i=1}^{n}\left(\left\|B_{i}\right\|+\left\|C_{i}\right\|\right)^{p} .
$$

In particular, if $B \in \mathbb{B}\left(\mathscr{H}_{2}, \mathscr{H}_{1}\right)$ and $C \in \mathbb{B}\left(\mathscr{H}_{1}, \mathscr{H}_{2}\right)$, then

$$
\omega\left(\left[\begin{array}{cc}
0 & B \\
C & 0
\end{array}\right]\right) \leq \frac{1}{2}(\|B\|+\|C\|) .
$$

Acknowledgement. The first author would like to thank the Tusi Mathematical Research Group (TMRG). 


\section{REFERENCES}

1. A. Abu-Omar and F. Kittaneh, Estimates for the numerical radius and the spectral radius of the Frobenius companion matrix and bounds for the zeros of polynomials, Ann. Func. Anal. 5 (2014), no. $1,56-62$.

2. A. Abu-Omar and F. Kittaneh, Numerical radius inequalities for $n \times n$ operator matrices, Linear Algebra Appl. 468 (2015), 18-26.

3. Y. Al-manasrah and F. Kittaneh, A generalization of two refined Young inequalities, Positivity 19 (2015), no. 4, 757-768.

4. J.C. Bourin, Matrix subadditivity inequalities and block-matrices, Internat. J. Math. 20 (2009), no. $6,679-691$.

5. P.R. Halmos, A Hilbert Space Problem Book, 2nd ed., springer, New York, 1982.

6. O. Hirzallah, F. Kittaneh and K. Shebrawi, Numerical radius inequalities for certain $2 \times 2$ operator matrices, Integral equations Operator Theory 71 (2011), 129-149.

7. F. Kittaneh, Notes on some inequalitis for Hilbert space operators, Publ. Res. Inst. Math. Sci. 24 (2) (1988), 283-293.

8. F. Kittaneh, A numerical radius inequality and an estimate for the numerical radius of the Frobenius companion matrix, Studia Math. 158 (2003), 11-17.

9. K.E. Gustafson and D.K.M. Rao, Numerical Range, The Field of Values of Linear Operators and Matrices, Springer, New York, 1997.

10. G. Popescu, Unitary invariants in multivariable operator theory, Mem. Amer. Math. Soc. 200 (2009), no. 941.

11. M. Sattari, M.S. Moslehian and K. Shebrawi, Extension of Euclidean operator radius inequalities, Math. Scand. (2016) in press, arXiv 1502.00083.

12. K. Shebrawi and H. Albadawi, Numerical radius and operator norm inequalities, J. Inequal. Appl. 2009, Art. ID 492154, 11-pp.

13. A. Sheikhhosseini, M.S. Moslehian and Kh. Shebrawi, Inequalities for generalized Euclidean operator radius via Young's inequality, J. Math. Anal. Appl. 445 (2017), no. 2, 1516-1529.

14. T. Yamazaki, On upper and lower bounds of the numerical radius and an equality condition, Studia Math. 178 (2007), 83-89.

${ }^{1}$ Department of Mathematics, Faculty of Mathematics, University of Sistan and BALUChESTAN, ZahedAN, I.R.IRAN.

E-mail address: mojtaba.bakherad@yahoo.com; bakherad@member.ams.org

22Department of Mathematics, Al-Balqa' Applied University, Salt, Jordan.

E-mail address: khalid@bau.edu.jo; shebrawi@gmail.com 